\title{
Rationale for short course primaquine in Africa to interrupt malaria transmission
}

\author{
Alice C Eziefula ${ }^{1}$, Roly Gosling ${ }^{2}$, Jimee Hwang ${ }^{2,3}$, Michelle S Hsiang ${ }^{2,4}$, Teun Bousema ${ }^{1}$, Lorenz von Seidlein ${ }^{5}$ and \\ Chris Drakeley ${ }^{1 *}$ on behalf of the Primaquine in Africa Discussion Group
}

\begin{abstract}
Following the recent successes of malaria control in sub-Saharan Africa, the gametocytocidal drug primaquine needs evaluation as a tool to further reduce the transmission of Plasmodium falciparum malaria. The drug has scarcely been used in Africa because of concerns about its safety in people with glucose-6-phosphate dehydrogenase (G6PD) deficiency. The evidence base for the use of primaquine as a transmission blocker is limited by a lack of comparable clinical and parasitological endpoints between trials. In March 2012, a group of experts met in London to discuss the existing evidence on the ability of primaquine to block malaria transmission, to define the roadblocks to the use of primaquine in Africa and to develop a roadmap to enable its rapid, safe and effective deployment. The output of this meeting is a strategic plan to optimize trial design to reach desired goals efficiently. The roadmap includes suggestions for a series of phase 1,2,3 and 4 studies to address specific hurdles to primaquine's deployment. These include ex-vivo studies on efficacy, primaquine pharmacokinetics and pharmacodynamics and dose escalation studies for safety in high-risk groups. Phase 3 community trials are proposed, along with Phase 4 studies to evaluate safety, particularly in pregnancy, through pharmacovigilance in areas where primaquine is already deployed. In parallel, efforts need to be made to address issues in drug supply and regulation, to map G6PD deficiency and to support the evaluation of alternative gametocytocidal compounds.
\end{abstract}

Keywords: Plasmodium falciparum, Malaria, Primaquine, 8-aminoquinoline, Transmission, Gametocyte, Glucose-6phosphate dehydrogenase deficiency, G6PD, Africa

\section{Background}

Current World Health Organization (WHO) guidelines recommend the "addition of a single dose of primaquine (PQ) $(0.75 \mathrm{mg} / \mathrm{kg})$ to artemisinin-based combination therapy (ACT) for uncomplicated falciparum malaria as an anti-gametocyte medicine, particularly as a component of a pre-elimination or an elimination programme" [1]. However, unlike recommendations for other anti-malarial treatments this does not come with the supporting statement "Strong recommendation, high quality evidence". This is because there are limited data to suggest that primaquine is safe and efficacious for this use, especially to support regulation and licensure. This is striking given that primaquine has been in the anti-malarial drug arsenal since the 1950 s and historical studies strongly

\footnotetext{
* Correspondence: chris.drakeley@lshtm.ac.uk

'Malaria Centre, London School of Hygiene \& Tropical Medicine, Keppel St, London WC1E 7HT, UK

Full list of author information is available at the end of the article
}

suggest that primaquine is highly effective at blocking transmission. Worldwide, 20 countries include primaquine as first-line treatment for Plasmodium falciparum in their national policy. None of these countries are in Africa [2].

There are an increasing number of reports of declining transmission intensity in many parts of sub-Saharan Africa, bringing malaria transmission to pre-elimination levels in some countries. There is also increasing recognition that additional strategies aimed specifically at the transmission stages of $P$. falciparum are required both to further reduce transmission and to sustain the gains made by current control efforts. The previously high levels of malaria transmission may be one of the main reasons why primaquine has not been used widely in Africa, with only very frequent delivery of the drug being likely to have any impact on transmission [3]. However, the most likely reasons for the limited use of primaquine in Africa are concerns over safety, given the conservation 
of the glucose-6-phosphate dehydrogenase (G6PD) deficiency polymorphism in the population.

Using an anti-malarial drug with the goal of interrupting malaria transmission rather than clinical cure necessitates a clearly-defined assessment of safety and efficacy with benefits at the individual level and at the community level being considered. For primaquine, the optimal dose to achieve such endpoints remains undetermined. The recommended $0.75 \mathrm{mg} / \mathrm{kg}$ dose is associated with significant haemolysis in some susceptible individuals [4-6], but this dose may well be excessive for the transmission-blocking activity [7]. For the purpose of comparison, doses in this report that are expressed as a milligram per kilogram $(\mathrm{mg} / \mathrm{kg})$ equivalent assume an average adult weight of 60 kilograms.

The limited safety data available on single dose primaquine has lead to the requirement of prior testing for G6PD deficiency and pregnancy to avert risk. The necessity for this additional testing has a significant impact on the feasibility, cost effectiveness and the achievable population coverage of large scale primaquine-based interventions. More information on the consequences of single-dose primaquine administration on individuals/ populations with a relevant range of G6PD enzyme activity levels is required urgently if 8 -aminoquinolines are to be deployed to interrupt transmission.

\section{Meeting objectives}

With these issues in mind, a meeting of experts was convened to review and discuss existing data on the use of primaquine in Africa for transmission-blocking and to examine the road-blocks that could be overcome to enable and inform its safe use.

Specific objectives of the meeting were to:

1. Identify key road-blocks to deployment of short course primaquine or similar drugs in Africa to reduce transmission of falciparum malaria.

2. Reach consensus on study endpoints so as to maximize comparability between transmission prevention studies.

3. Generate a list of deliverables that will move forward deployment of primaquine in Africa.

\section{Meeting sessions}

\section{Country program perspectives and potential use for primaquine}

Chris Drakeley and Roly Gosling introduced the meeting by providing the current context for the use of primaquine and highlighting the fact that the reductions in malaria transmission that have been described in many sub-Saharan African settings may well be linked to increasing spatial, temporal and even demographic heterogeneity in infections. Spatial targeting of control efforts is likely to make interventions, such as mass drug administration (MDA) more feasible [8]. National malaria control programmes that have seen success in malaria control in the last decade are looking to implement new tools to sustain existing reductions and to further reduce transmission. The question is whether primaquine is one of these tools?

Salhiya Ali described current malaria transmission in Zanzibar, which is characterized by perennial and declining transmission. The sporozoite rate decreased from 4.3 in 2005 to $0 \%$ in 2009 and the most recent parasite prevalence was $0.067 \%$. Recent Zanzibar Malaria Control Programme reports suggest that transmission has become highly heterogenous with cases restricted to relatively few weeks per year and to a few localities. Primaquine is not used, but its use could be considered to facilitate further reductions by targeting hot spots, or in treating confirmed clinical cases. The local distribution of gametocytaemia and G6PD deficiency is not known.

In Ethiopia, both P. falciparum and Plasmodium vivax are endemic and Ashenafi Assefa indicated that the malaria strategy for 2011-2015 includes a plan for elimination by 2020 . Primaquine was used in Ethiopia for 25 years up until 1990. Chloroquine (CQ) plus primaquine was firstline treatment for both species. There is no documentation of adverse effects due to primaquine in this period. When sulphadoxine-pyrimethamine (SP) was introduced, it was considered not feasible to administer three drugs, therefore, primaquine was dropped. At present, primaquine is used for radical cure of $P$. vivax, but not for $P$. falciparum. The barriers to using primaquine in Ethiopia include: 1) a lack of documentation of the distribution and clustering of G6PD deficiency (small studies suggest that prevalence is between 1.4 and $6.7 \%$ among some minority groups) [9], and 2) uncertainty about the efficacy of primaquine for interrupting transmission of P. falciparum in Ethiopia.

Karen Barnes gave a historical perspective of malaria control in South Africa. Previously, the country had high levels of malaria transmission. In 1938, there were 22,000 deaths due to malaria in Kwazulu-Natal. Subsequently, an aggressive approach to malaria control including mapping, malaria surveys, and vector control has reduced the burden considerably but case incidence has remained at a steady state since 2001. The Ministry of Health has now set a goal for elimination by 2018. The biggest challenges include imported malaria, and the perception that malaria is not a public health problem, leading to central budget cuts. Given the already aggressive measures in place, the addition of a transmission-blocking drug such as primaquine could be required to achieve elimination. One challenge is that primaquine is only available on an individual patient basis for radical cure of $P$. vivax. In 
South Africa, the very high rate of tuberculosis and HIV infection means that the potential for drug interactions with other anti-infective therapies must be considered if primaquine is to be used at a population level. The risk of primaquine-associated haemolysis in people living with HIV infection may differ from that in uninfected people.

In contrast to the aforementioned countries, Diadier Diallo reported that malaria transmission in Burkina Faso is still high. The use of a combination of interventions, such as long-lasting insecticidal nets (LLINs), indoor residual spraying (IRS), and effective artemisinin combination therapy (ACT) with a long half-life partner drug such as dihydroartemisinin-piperaquine is a proposed strategy. Co-administration of ACT with primaquine (or alternatives such as methylene blue) for confirmed malaria episodes and mass drug administration (MDA) may help to further reduce transmission. This strategy may be particularly appropriate in the Sahel area where transmission is highly seasonal and relatively low, making it a potential target for elimination activities. Challenges include the high mobility of human and vector populations particularly from Mali and Niger.

\section{Historical studies on single dose or short course primaquine for blocking transmission of $P$. falciparum}

Chi Eziefula highlighted that the current recommendations for primaquine are based on studies with very small numbers of participants. The parent 8-aminoquinoline, pamaquine (or plasmoquine), developed in the 1920s, was shown to have activity against $P$. vivax and Plasmodium ovale relapses, and against both sporozoites and gametocytes of all species [10,11]. A derivative of pamaquine, primaquine was developed in the 1940s by the United States army to prevent relapse of $P$. vivax in soldiers returning from Korea and to prevent the import of malaria into the country [12].

In 1973, the WHO recommended a single dose of primaquine $(0.75 \mathrm{mg} / \mathrm{kg})$ for malaria transmissionblocking and considered prior screening for G6PD deficiency unnecessary [13]. It was not until 2010 that the WHO Malaria Treatment Guidelines (Second Edition) changed to indicate that the risks of haemolysis in G6PD deficient patients should be given consideration prior to primaquine-based interventions.

The currently recommended single dose of primaquine is based on limited efficacy data. In 1961, in Liberia, Burgess and Bray found that a single dose of 0.75$1.5 \mathrm{mg} / \mathrm{kg}$ primaquine administered to12 children cleared circulating gametocytes by day 9 [7]. In 1961, also in Liberia, Gunders administered $0.45-1.1 \mathrm{mg} / \mathrm{kg}$ of primaquine in combination with pyrimethamine to 22 children and adults. Gametocytes were cleared after a mean of 5 days post treatment, and no mosquito infections occurred in feeding assays [14]. Primaquine was paired with amodiaquine (AQ) in a large scale MDA conducted by Clyde in 1962 in a hyperendemic area of Tanzania. More than 15,000 subjects were studied in three clusters: weekly administration, fortnightly administration, and monthly administration. Outcome measures included asexual parasite, gametocyte and sporozoite rates. After six months there was a ten-fold reduction in parasite prevalence with weekly and fortnightly administration but not with monthly administration [3]. Except for the work by Clyde, there are no substantial field data that indicate that single dose primaquine decreases transmission of $P$. falciparum.

Safety data for primaquine use in Africa or African Americans are equally limited despite the fact that they inform contemporary guidelines. Burgess and Bray comment that primaquine was "well-tolerated"[7]. Clyde reported no safety data and it is unclear who was excluded from treatment [3]. In a series of studies in G6PD deficient African-American volunteers, Alving and colleagues showed that, in three individuals, haemolysis occurred with daily administration of 30mg (approximately $0.5 \mathrm{mg} / \mathrm{kg}$ ) of primaquine. But, after three weeks, the haematocrit recovered and lower doses resulted in less haemolysis. Eight weekly doses of $60 \mathrm{mg}$ and $45 \mathrm{mg}$ were not associated with haemolysis $[15,16]$. Daily administration of $30 \mathrm{mg}$ of primaquine to African Americans resulted in significant haemolysis in 1\%, compared to no severe haemolysis when $15 \mathrm{mg}$ was administered [17]. Tolerance in a pregnant woman (28 weeks gestation) has only been reported by Burgess and Bray, but there was no documentation of birth outcomes [7]. In a more recent study, Kenyan school children were randomized to receive $15 \mathrm{mg}$ primaquine daily or three times a week as a malaria prophylactic. It is not clear whether G6PD deficient individuals were included and haemoglobin levels are not reported but again the authors note simply that "primaquine was remarkably well tolerated in our studies" [18].

Kevin Baird remarked that any discussion about primaquine efficacy is necessarily also a discussion about toxicity as there are inherent risks of the drug in situations when the individual patient may not benefit. $\mathrm{He}$ highlighted the importance of employing the ethical principles of autonomy, justice and beneficence to gametocytocidal therapy [19]. The $45 \mathrm{mg}$ dose of primaquine is based on data obtained in very few, healthy individuals. This dose was proposed in an era where the goal of the US military was not to find the lowest efficacious dose, but rather to show that the drug worked. The first dose-finding study by Alving in 1960 included one single patient [16]. It was subsequently observed that daily but not weekly administration of $0.25 \mathrm{mg} / \mathrm{lb}$ of body weight $(\sim 0.55 \mathrm{mg} / \mathrm{kg})$ to G6PD deficient-children resulted in 
haemolysis [20]. Rieckmann and Burgess both showed declines in gametocytes, oocysts and sporozoites following a dose of $45 \mathrm{mg}$ of primaquine but a similar efficacy was seen with lower doses of $30 \mathrm{mg}$ and $15 \mathrm{mg}[7,21,22]$. Importantly, these evaluations were conducted without co-administration of a blood schizontocidal drug.

In 1944, the US government abandoned pamaquine as a means of preventing relapses of $P$. vivax due to its haemolytic toxicity and drug interactions. Primaquine was introduced as a gametocytocidal agent at the $45 \mathrm{mg}$ dose based on Alving's work, a dose which was readily available and in use for chemoprophylaxis in American soldiers in Southeast Asia at the time. Some significant haemolysis was seen, mostly in African Americans; there were no deaths but there were several cases of renal failure with daily dosing for 14 days[17]. Summarily, the recommended $45 \mathrm{mg}$ dose may be too dangerous for use in mass drug administration, especially given the limited data on transmission reduction with this strategy.

\section{Recent studies on the use of primaquine in Africa}

Data from two Tanzanian studies which employed single dose primaquine were reviewed by Teun Bousema. In the first study, treatment with sulphadoxine-pyrimethamine (SP) and artesunate (As) was given to children aged 3 to 15 years with uncomplicated falciparum malaria. They were randomized to receive placebo or a single dose of $0.75 \mathrm{mg} / \mathrm{kg}$ of primaquine on the third day of treatment (day 2). Compared to the control arm, primaquine administration on day 2 decreased the area under the curve of gametocyte density over time and the duration of gametocyte carriage. The effect was apparent for two weeks; using quantitative real time nucleic acid sequencebased amplification (QT NASBA), 3.9\% had gametocytes on day 14 in the primaquine arm, and the density was extremely low, compared to a prevalence of $62.7 \%$ in the control arm [23]. Haemoglobin fell in both arms but the drop was more pronounced in the primaquine arm. However, this effect was transient and there was no symptomatic anemia. A haemolytic effect was seen even in some individuals without genotypic (A- variant) G6PD deficiency [23].

In a subsequent cluster randomized study, using MDA in lower Moshi [24], single dose primaquine was given with SP plus As treatment to 1110 individuals older than 1 year with primaquine dosages based on weight (approximately $0.75 \mathrm{mg} / \mathrm{kg}$ ). It was not possible to assess post-intervention incidence or prevalence because $P$. falciparum transmission had dropped to very low levels. However, safety outcomes, based on haemolysis, were available. Moderate haemolysis occurred following primaquine treatment in $40 \%$ of G6PD deficient (A- genotype) individuals but in only $4.5 \%$ of nondeficient individuals. There was no clinical compromise due to anemia in any of the children, except in one child in the primaquine arm, whose haemoglobin dropped from $8.3 \mathrm{~g} / \mathrm{dL}$ to $4.8 \mathrm{~g} / \mathrm{dL}$. It was noted that in all cases haemolysis was transient, recovering by day 14 after treatment.

As a former colleague of Professor Li Guoqiao, Keith Arnold represented him and presented data from an MDA campaign in Moheli Island, Comoros. Dr. Arnold began by reviewing Professor Li's work on primaquine in South East Asia, which served as the basis for the drug regimen used in Comoros. In the late 1990s, Professor $\mathrm{Li}$ developed CV8 (320 mg piperaquine phosphate, $32 \mathrm{mg}$ dihydroartemisinin, $5 \mathrm{mg}$ primaquine phosphate, $90 \mathrm{mg}$ trimethoprim). An estimated 1.3 million doses of this drug were administered across Vietnam as part of the National Malaria Control Programme in 2000. There were no documented reports of haemolysis. Data were presented from subsequent dose-finding studies. Artequick (dihydroartemisinin piperaquine given at 0 and 24 hours) was administered in clinical cases followed as inpatients for 30 days followed by administration of $6 \mathrm{mg}$ (7 patients), $7.5 \mathrm{mg}$ (3 patients) or $8 \mathrm{mg}$ (32 patients) of primaquine. A $7.5 \mathrm{mg}$ dose of primaquine rendered gametocytes non infectious at 24 hours. Following $8 \mathrm{mg}$ of primaquine, there were oocysts but no sporozoites in membrane-fed mosquitoes. He decided on the use of Artequick $+9 \mathrm{mg}$ primaquine for MDA after performing safety studies using $8 \mathrm{mg}$ and $10 \mathrm{mg}$ doses in small numbers of individuals with G6PD deficiency in South East Asia. An MDA campaign in 2003 in Cambodia using this regimen resulted in a large reduction in population parasite carriage over three years [25].

In Moheli Island, Comoros the baseline P. falciparum parasite prevalence in children ranged from 10-95\% in 25 villages. Given a mosquito life expectancy of 30 days, the strategy was to give Artequick for three days plus $9 \mathrm{mg}$ of primaquine on day 1 (Round 1) and day 35 (Round 2). Also, beginning on day 21, $9 \mathrm{mg}$ primaquine alone was given every 10 days, 12 times. Patients less than six months of age were excluded. Treatment coverage for both rounds was reported as $>90 \%$ and data from monitoring between 2007 and 2009 suggested a reduction of parasite prevalence to $<5 \%$. The exception was an area on the south of the island where parasite rates decreased from $94 \%$ to $19 \%$ with frequent migration from a nearby island suggested as the reason for the persistence of parasites. There were no reports of haemolysis, although it was not measured objectively. The baseline prevalence of G6PD deficiency was estimated to be $15 \%$.

\section{G6PD deficiency prevalence testing and safety issues}

G6PD is an essential erythrocytic enzyme. G6PD deficiency is one of world's most common genetic polymorphisms. Dennis Shanks described the current array 
of diagnostic tests available to test for G6PD deficiency. Testing of the enzymatic activity of G6PD on freshlycollected blood samples is the most widely used method. The NADPH fluorescent spot test is most commonly used and is currently recommended by the International Committee for Standardization in Haematology, but it requires a UV lamp and is difficult to do on high volumes of samples. Other diagnostic tests include cytochemical assays, DNA sequence analysis of the G6PD gene, and some rapid diagnostic test formats not yet validated for public health application. In theory, testing for G6PD deficiency is not difficult, but most tests have limitations for large-scale field application, such as expense, requirement for electricity, duration of test procedure, and sensitivity of reagents to light and heat, low detection threshold, and relatively low throughput capacity.

Rosalind Howes described G6PD deficiency as being widespread in tropical regions of sub-Saharan Africa, commonly affecting over $15 \%$ of the male population, and in some isolated areas of West and Central Africa reaching up to $30 \%$ of the male population. It is considered that severe G6PD deficiency is likely to exist in Africa but its prevalence is unknown. Shanks noted that country-wide MDA with primaquine has been used in China and Nicaragua, both areas with a low prevalence of G6PD deficiency and that in both programmes there were some cases of severe haemolysis. The three primary safety/ tolerability issues with primaquine are gastrointestinal upset, methaemoglobinaemia, and haemolytic anemia in those who are G6PD deficient. G6PD enzyme activity is at best a partial biomarker of clinical effect and the clinical effect is likely dependent on other factors including red blood cell count, gender, and other genetic factors.

\section{Testing for G6PD deficiency}

Gonzalo Domingo observed that genotyping for G6PD deficiency is most commonly carried out for known prevalent mutations at the risk of misclassifying study participants with unknown G6PD deficiency traits as normals. Phenotyping, either quantitative or qualitative, determines G6PD activity in red blood cells and can be defined as a relative deficiency in activity compared to a predefined "normal" activity or in absolute terms in units per gram of haemoglobin. Most studies in Africa have used a semi-quantitative/qualitative fluorescent spot test and observed a high degree of discordance between phenotyping and genotyping not limited to just heterozygous women. Other phenotypic tests e.g. cytochemistry can identify heterozygous females. Spectrophotometry is the gold standard and fluorescent spot tests are useful for screening. The ideal specification for a G6PD deficiency test is difficult to achieve as there is no defined acceptable cut-off of G6PD activity. The challenges are that the measurement of enzyme activity is extremely sensitive to temperature, specimen volume, and possibly specimen type. Of the available tests that run on point-of-care platforms, BinaxNOW is limited by its operating temperature and Access Bio by its small sample volume, which may be a source for performance variability. The BinaxNOW test detects a cut-off of $30-40 \%$ enzyme activity and was designed to detect hemizygous males. Detecting heterozygous females require platforms that can detect and enumerate intraerythrocytic G6PD activity. The next steps include an evaluation of currently available tests for G6PD deficiency under ideal laboratory conditions, field evaluation under controlled conditions, and engaging with the diagnostic sector to define a value proposition for pointof-care G6PD deficiency tests. Ongoing efficacy studies for primaquine represent an opportunity to obtain G6PD deficiency cut-off levels.

\section{Examples of possible study designs - clinical and field-based}

Lorenz von Seidlein and Teun Bousema considered the sequence of studies required to establish the role of primaquine in the response to artemisinin resistance as well as for the elimination of falciparum malaria. Before population-level interventions are considered, three main questions will need to be addressed: 1) What drug concentration is needed to inhibit gametocytes, 2) which primaquine regimen is required to achieve these gametocyte inhibitory concentrations and 3) can this dose be safely administered to both sexes and all age groups? Excluding young children and women of reproductive age from MDA will seriously reduce coverage and is likely to render any intervention meaningless. Since a prospective study of giving single dose primaquine during pregnancy is not likely to be approved, retrospective approaches e.g. pharmacovigilance during large field trials should be explored as a way of gaining information about the safety of primaquine in pregnancy.

One option for field evaluation is the cluster randomized trial. A double-blinded community-randomized, placebo-controlled trial in The Gambia evaluated MDA with sulphadoxine- pyrimethamine (SP) plus single dose artesunate (AS1) in 18 villages and achieved $89 \%$ coverage [26]. There was an initial decrease in malaria incidence but the effect quickly disappeared. Possible reasons for a failure to reduce transmission intensity might be that the baseline transmission intensity was too high, that there was migration of infected individuals or mosquitoes, or that the drug regimen was not ideal. A doubleblinded community-randomized, placebo-controlled trial was conducted in Tanzania in a setting of very low and 
seasonal malaria transmission (entomological inoculation rate of approximately 2) using MDA with SP on day 1 plus artesunate for 3 days and primaquine on day 3 [27]. Coverage of $93 \%$ was achieved, but the study failed to show a reduction in transmission intensity due to the small number of outcome events (P. falciparum infections) in both the intervention and control groups. These studies raise two important questions: 1) Are sub-microscopic parasite densities sufficient to sustain transmission and 2) what is the ideal transmission intensity at which to conduct MDA? It was considered that studies designed to detect the community benefit of ACT versus ACT plus primaquine would potentially necessitate very large sample sizes and alternative strategies to evaluate MDA should also be considered. The community effect of insecticidetreated bed nets extends beyond the households that use nets and has been estimated by measuring the distance between control and intervention villages and compounds where protection is seen. Such an effect may exist for primaquine- based interventions such that targeted coverage has a high impact. Less ambitious trial designs could encompass treatment of clinical malaria cases, focal screen-and-treat campaigns, or primaquine could be incorporated into active case detection activities using standardized outcome measures such as entomological parameters, gametocyte prevalence by molecular methods, parasite prevalence/ molecular force of infection, and malaria incidence during follow-up.

The design of trials of MDA with primaquine should help inform a potential strategy for interventions. What is the threshold endemicity level at which MDA with primaquine should be considered? How many rounds of MDA are required and at what interval to give a given effect? Even if efficacy and safety can be established, the issue of willingness to participate in MDA must be considered. In settings of very low transmission and minimal risk, e.g. Swaziland, the community might not be as accepting of MDA as compared to a country with higher endemicity as the perceived benefit is lower.

\section{Potential study endpoints-clinical and field studies}

Heiner Grueninger emphasized that study endpoints should be designed to facilitate both effective treatment and increased knowledge of the study drug. In the context of using primaquine for a new indication of transmission-blocking, the study design should address the requirements set by authorities for obtaining regulatory approval to use the drug. Consequently, endpoints should be considered with input from both industry and policy makers in order to expedite drug deployment in endemic settings.

Chris Drakeley discussed biomedical efficacy endpoints. Abrogation of infection in mosquito infectivity studies is a compelling functional bioassay yet only one existing study involving primaquine satisfied Cochrane review criteria (Graves and Gelband, in press). In this study, mefloquine and SP plus primaquine stopped infection over 14 days post treatment [28]. The mosquito feeding assay methods for assessing post treatment infectivity of subjects offer different options for evaluation, but are not standardized. Direct skin feeding of mosquitoes on treated individuals is most representative of natural infection dynamics but presents logistical and ethical concerns. Using venous blood allows both direct membrane feeding but also serum replacement with untreated or treated serum to examine the effect of different serum compositions, such as drug metabolites. Reproducibility of results is an important issue with no clear guidelines on how to feed mosquitoes, how many mosquitoes should be fed per assay, because the robustness of the estimate of prevalence of infection depends on the number fed [29], and on which day post-treatment participants should be tested for infectivity. For example, primaquine has a short half-life so infectivity could be measured after 24 hours, whereas, for the purpose of MDA, it is probably pertinent to know for how long the subject has reduced infectivity and testing for infectivity up to 28 days may be relevant. This latter point could be addressed by staggering sampling time points between participants to reduce the number of bleeds per individual. Further studies may be required to confirm the effect on infectiousness to wild mosquito populations as natural infections have been shown to be successful at very low gametocyte densities suggesting high vector susceptibility [30]. Such feeding experiments may not be warranted or practical for larger field evaluations and a surrogate marker for transmission would be preferable.

Although, there is no standardized, validated marker of infectiousness of the human host, the most widely used marker to compare drugs is the prevalence of gametocytes 7 days post treatment [31]. Gametocyte density is less relevant at low gametocyte counts found in chronic and asymptomatic infections as the correlation between infectivity and low gametocyte density is poor. The measurement of gametocyte prevalence and density depends on the method of detection with 5- to 10- fold differences seen with molecular methods compared to microscopy[32]. Gametocyte densities can be integrated using area under the curve (AUC) to provide an estimate of gametocyte carriage [33,34]. In natural infections this is likely to vary by age with young children with clinical disease having short, intense gametocytaemia (abrogated by drugs or gametocyte death) and older semi-immune individuals, who can have asymptomatic infections for up to a year [35] and maybe longer, with a more prolonged AUC.

The issue of how to tailor the design of studies using primaquine to include endpoints that are meaningful to 
regulatory authorities was tackled by Justin Green. The key question is what level of evidence do we require in order to use primaquine as a transmission-blocking agent? He referred to ongoing studies using tafenoquine to highlight how bespoke endpoints are being used to achieve licensure. Tafenoquine is an 8-aminoquinoline developed by the US army and the Walter Reed Army Institute of Research (WRAIR) with GlaxoSmithKline (GSK). It has a long half-life (14-17 days), which may confer advantages as an anti-parasitic agent, but also risks, given that the duration of haemolysis in individuals with G6PD deficiency is also prolonged [36]. The drug is slowly metabolized and the parent compound is responsible for the anti-malarial effect [37]. Tafenoquine is being developed as a radical cure of $P$. vivax infection. Green described a dose-ranging study in individuals over 16 years with $P$. vivax infection evaluating chloroquine alone compared with standard dose chloroquine plus primaquine $15 \mathrm{mg}$ (for 14 days) and different single doses of tafenoquine (50mg, 100mg, 300mg, and 600mg) given on day 1 or day 2 (NCT01376167). The primary endpoint is relapse at 6 months with secondary endpoints of relapse at 4 months, time to relapse, parasite clearance time, fever clearance time, gametocyte clearance time (by microscopy), safety and pharmacokinetics/ pharmacodynamics.

These pivotal endpoints are designed with regulatory requirements in mind so that wording related to endpoints can be incorporated into a label claim. From the perspective of industry, this can determine the potential volume of sales (the percentage of the primaquine market obtainable). For trials with primaquine, or other transmission-blocking candidates, it is necessary to decide how important it is that the study endpoint is on the label and whether stakeholders demand a "label claim" or an indication for approval. For transmission markers to stand as endpoints for a regulatory level trial, one would need validation that the marker, e.g., a
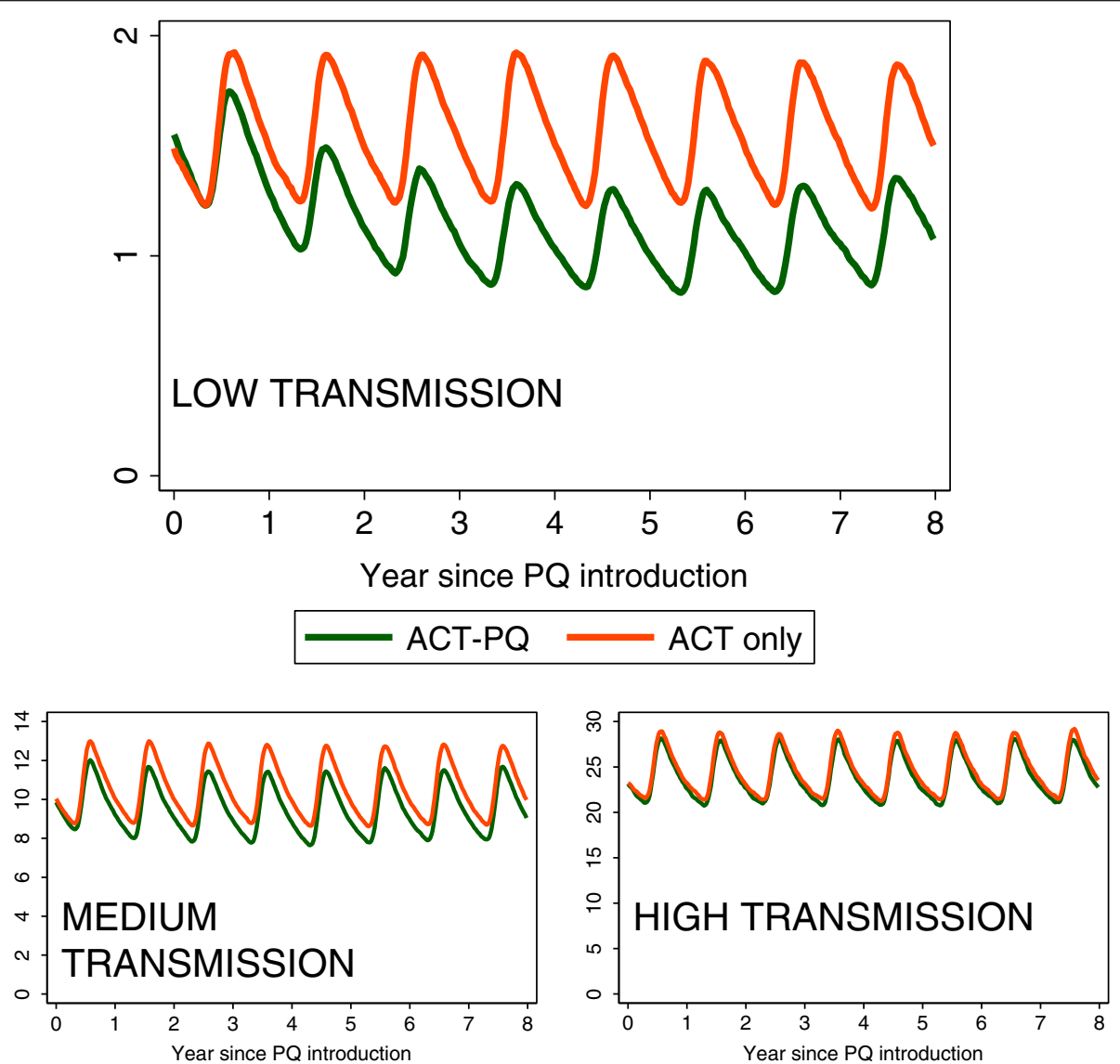

Figure 1 Preliminary modeling of administration of primaquine together with ACTs in a range of transmission settings. A simulation of adding primaquine to ACT first line treatment versus ACTs only in a seasonal setting. In this simulation $80 \%$ of clinical cases are treated with ACT alone or ACT-PQ. Primaquine is assumed to reduce the duration of infection by $78 \%$ and the level of infectiousness by $67 \%$ in treated patients compared to those treated with ACT alone. In a low transmission scenario, adding PQ to the treatment of clinical cases causes a higher relative reduction than in higher transmission scenarios. Ro differs between settings. Migration is not allowed for. With the kind permission of Lucy Okell, Jamie Griffin \& Azra Ghani. For further details, see reference [40]. 
molecular method such as detection of pfs 25 with QT NASBA [38] or microscopic gametocytaemia, correlates with transmission.

Typically, the pharmaceutical industry focuses on the risk-benefit of a particular drug in the individual. For primaquine, the drug may be of more benefit to someone other than the recipient, raising the ethical question of whether it is acceptable to give a drug for community benefit. This issue is also pertinent to transmissionblocking vaccines [39]. Justin Green considered that it is crucial that primaquine trials include individuals with G6PD deficiency (including heterozygote females) and describe the risk of haemolysis in these patients. There is no consensus on whether there is any acceptable degree of haemolysis following a drug intervention for malaria in clinical cases or in asymptomatic individuals.

\section{Modeling the potential use of primaquine}

Teun Bousema discussed how to extrapolate the effect of primaquine in the individual to community-level transmission, acknowledging that the infectious reservoir of malaria may vary with transmission setting. A recent model by Lucy Okell and colleagues [33] suggests that infectiousness post ACT alone is 13 days and post ACT plus primaquine is 3 days. Using this model that incorporates population age structure, immunity, heterogeneous exposure and as well multiple interventions as covariates, the addition of primaquine to $\mathrm{ACT}$ as first-line treatment significantly reduces transmission in low endemic settings but not in higher transmission settings (Figure 1). The proportion of people who received primaquine in addition to ACT is a key parameter suggesting primaquine needs to be given with all courses of ACT to have an effect. The models were further extended to investigate the effect of primaquine as part of an MDA [40] in a non-seasonal setting with $9 \%$ prevalence of $P$. falciparum. Giving MDA every four months caused an $80 \%$ reduction in transmission, but not elimination. With MDA every six weeks one could plausibly reach elimination. Preliminary models suggest that MDA may be more successful in areas of seasonal transmission (Figure 2). The duration of drug action is important and a long acting ACT plus a long acting 8-aminoquinoline could be an optimal combination.

An approach targeting malaria transmission hotspots may be appropriate for all endemic settings [8]. The hypothesis is that hot spots catalyse transmission and targeting them would reduce transmission both within and outside the hotspot. Modeling hotspot interventions with no drug treatment but with insecticide-treated bed nets scaled up to $80 \%$ coverage and targeted IRS had a significant effect on transmission. The effect of adding primaquine should be investigated. Models of transmission assume a long time-course and there was discussion

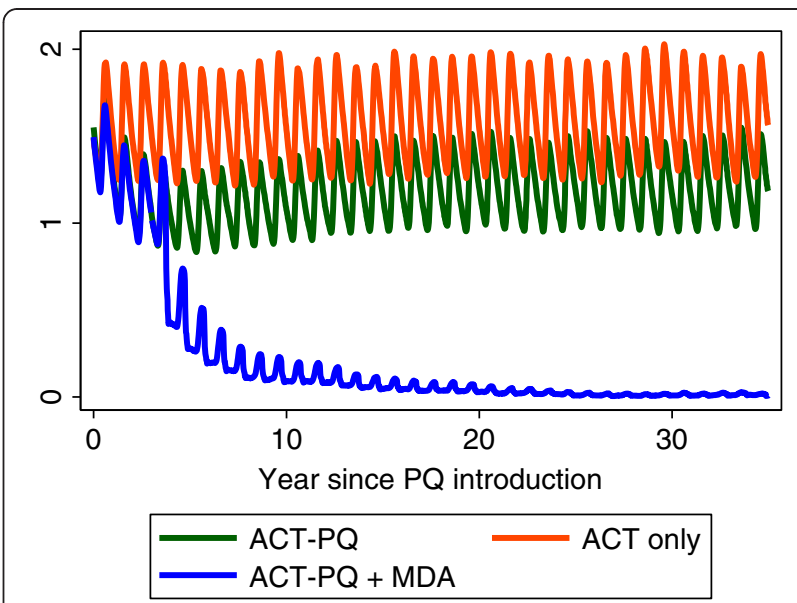

Figure 2 The effect of annual MDA with primaquine in addition to primaquine and ACT treatment of clinical cases in a low transmission setting. In a seasonal, low transmission setting, giving $\mathrm{ACT}+$ primaquine to clinical cases plus an annual MDA with $\mathrm{ACT}$ plus primaquine could reduce malaria levels close to elimination if repeated for a number of years; however the model does not allow for immigration of malaria cases. With the kind permission of Lucy Okell, Jamie Griffin \& Azra Ghani. For further details, see reference [40].

as to the stability of hot spots and how this would affect the efficacy of an intervention.

\section{Meeting outputs}

Possible approaches for the use of primaquine to interrupt malaria transmission

Having reviewed the existing data, the second aim of the meeting was to identify the roadblocks to deployment of primaquine in Africa (Figure 3), decide on common study endpoints and to determine the next steps. As a starting point the group determined the intended indications of primaquine (Figure 4), a target product profile (Figure 5) and common endpoints for infectivity, efficacy and safety studies (Figure 6).

\section{Key roadblocks to the deployment of primaquine} Safety and efficacy of primaquine

The paucity of evidence for primaquine's safety and efficacy for transmission-blocking were seen as major issues, particularly, the lack of data supporting the safest and most efficacious dose. If primaquine is going to be used to maximal benefit then it must be safe to deploy in G6PD deficient individuals and women of childbearing age and it must be safe to co-administer with HIV and tuberculosis treatments without adverse drug interactions. Most crucially, evidence is lacking for any transmissionreducing effect in the community from the addition of 


\section{Summary of key discussion points-Roadblocks to the deployment of primaquine}

- Evidence gaps. The mechanism of gametocytocidal action of primaquine should be investigated in order to optimally measure its effect.

- Future trials should be standardised with consistent safety and efficacy endpoints and be conducted in a range of transmission settings.

- Gametocyte density measures should be calibrated with standardised membrane feeding to investigate the reduction in infectivity post intervention.

- A standardised and functional measurement of primaquine-associated haemolysis needs definition.

- Studies should be designed with a target product profile for an ideal transmission-blocking drug in mind. This must include safety in G6PD deficient individuals.

- There is insufficient data on population (and sub-population) prevalences of G6PD deficiency in countries where primaquine could potentially be deployed.

- G6PD deficiency diagnostics need agreed standards with relevance to primaquine associated haemolysis and should be usable in the community/ field setting.

- The target population and method of distribution for maximal effect need to be determined; is there a role for primaquine in treatment of clinical cases versus MDA in asymptomatic infected individuals, depending on the transmission intensity?

- Deciding when and where to deploy MDA is very important. Previous WHO MDA guidelines were for reducing prevalence from high to low levels. However, it was felt that a more appropriate use of MDA would be to reduce low prevalence to zero.

- Primaquine-based MDA or an equivalent community intervention should be used in conjunction with vector control measures (LLINs and IRS).

\section{Policy/implementation challenges}

- Important ethical issues arise with the administration of a drug with benefit primarily to the population rather than to the individual and these have implications for study design. Ultimately, an acceptable level of risk to the individual must be agreed upon.

- The interactions of primaquine with co-administered ACTs, antiretrovirals and antituberculosis medications should be explored.

- Stakeholders (ethical review boards, drug authorities, WHO) should be involved early to:

- optimise the translation of study outcomes to effective deployment.

- ensure long-term availability of quality-assured primaquine.

- ensure that regulatory requirements are satisfied; currently, malaria transmissionblocking is not an authorised indication for primaquine.

Figure 3 Summary of key discussion points_roadblocks to the deployment of primaquine.

primaquine to routine anti-malarial treatment of symptomatic individuals.

\section{Suitable endemicity for use of primaquine}

It was agreed that use of primaquine is most likely to have an impact on transmission intensity in areas characterized by low endemicity prior to the intervention, i.e. P. falciparum parasite rate (PfPR) by microscopy of less than $5 \%$, or an EIR (entomological inoculation rate) less than 1. In such settings, there is a low frequency of symptomatic parasitaemia so the greatest benefit is likely to result from treating asymptomatic infections as well, through MDA or screen-and-treat initiatives. The optimal strategy for delivering primaquine-based MDA in terms of who to treat, at what threshold endemicity, with what regimen and how often is unknown.

Mathematical modelling indicates a limited effect at higher transmission intensities (PfPR> 10\%). However, 
Primaquine efficacy for transmission studies

- Membrane feeding assay for mosquito infection rate i.e., prevalence of mosquitoes infected on day 7. Further validation of membrane feeding may be required to allow comparability between sites. Infectivity to be correlated with gametocyte prevalence \& density to provide proxy measures for efficacy in areas without membrane feeding capacity.

- Day 0 \& 7 gametocyte carriage and density by microscopy (minimum 200 fields on a thick film) and molecular methods. Area under the curve of gametocyte density over time with multiple time-points in the first two weeks (e.g. day $0,1,2,3,7,10,14$ ).

- Endpoints for assessing community-targeted primaquine delivery (e.g. MDA).

$\circ$ Incidence of infection

- Force of infection determined by molecular and serological methods

- Parasite prevalence by PCR

- An individual benefit needs to be defined based on these endpoints to guide discussions on what level of individual risk is acceptable

Safety

- Absolute decrease (proposed threshold of $2.4 \mathrm{~g} / \mathrm{dL}$ ), percentage decrease and rate of decrease in haemoglobin from baseline.

- Adverse event and tolerability monitoring for which there should be a minimum follow up duration of 28 days for safety and powering of sample size to include safety outcomes.

Figure 4 Endpoints for standardisation and regulatory compliance.

further iterations are needed to assess the additional effect of primaquine interventions together with other control tools at a range of transmission intensities. As was the situation in Aneityum, Vanuatu [41], there may be other higher transmission settings where interruption of transmission could occur using MDA with primaquine because of limited human migration.

\section{Partner drug for primaquine}

For community campaigns with primaquine, the partner ACT should probably differ from the recommended firstline anti-malarial treatment. An alternative ACT may be required for community-wide MDA or in circumstances where repeated rounds of MDA are envisaged. However, in smaller hotspots of high transmission intensity where fewer rounds of treatment with ACT-primaquine are needed, the standard first-line ACT could be considered as the partner to primaquine. The relative gametocytocidal activity of the partner ACT, its half-life for killing asexual parasites and the potential for drug interactions or for synergy with primaquine will need to be considered.

\section{Drug supply and regulation}

The manufacture and supply of the appropriate dose and formulation of primaquine was seen as a major obstacle for primaquine deployment. Currently, there are primaquine shortages globally and in Africa, the procurement of supplies to treat $P$. vivax where it is endemic is a challenge.

Further information on the current challenges for the manufacture and supply of single- or low-dose primaquine is required. A review of the current situation of primaquine manufacture and supply should be carried out with the aim of identifying the steps needed to ensure an adequate supply of primaquine formulated in the correct dose should low dose primaquine be found to be efficacious. It is likely that primaquine for the clearance of $P$. falciparum gametocytes will remain off label. In order to ensure the smooth process from manufacture to implementation, it was recommended that stakeholders from industry and governments, including regulatory authorities be brought together to discuss these challenges. 


\author{
A Target Product Profile for primaquine \\ A target product profile was developed by the group (see below) in order to support the planning of \\ necessary trials. \\ - Indications and usage - Transmission blocking agent for co-administration with ACTs. \\ - Safety - Maximum safety because no immediate benefit to individual, ideally safe in \\ pregnancy. The acceptable serious adverse effect rate is to be weighed against the number \\ of cases/ malaria morbidity events averted and the risks associated with prophylaxis. The \\ benefits to the individual should be considered as potential prevention of re-infection when \\ primaquine is appropriately deployed. There should be no adverse interactions with \\ antiretroviral or anti-tuberculosis therapy. \\ - Efficacy/Endpoint: High efficacy for blocking transmission to mosquitoes at day 7 and \\ reducing day 7 gametocyte carriage. \\ - Dosing regimen: co-administered with ACT. Primaquine given on either day 1, or day 3. \\ Simple oral dosing (available small-dose formulations), paediatric formulation. \\ - Cost: $<\$ 1$. \\ - Co-packaging or co-formulation with ACT preferred. \\ - Exclude from indication: \\ - Ideally no one. \\ - Fall back: G6PD deficient (including a pregnant women if she or the father of the \\ baby has G6PD deficiency). If there are exclusions, the added operational \\ considerations (G6PD deficiency test performance and cost, logistics and risks in \\ pregnancy) required to carry out the additional screening will need to be evaluated.
}

Figure 5 A Target Product Profile for primaquine.

\section{Alternatives to primaquine}

The meeting agreed that seeking alternative gametocytocidal drugs to primaquine was paramount due to the safety concerns with 8-aminoquinolines. The 8-aminoquinoline tafenoquine appears to have a similar safety profile to primaquine (haemolysis in people with G6PD deficiency), but being long-acting, may potentially inhibit gametocyte infectivity for longer. Should a safe, low dose be found, tafenoquine could be a useful tool in the elimination of P. falciparum. There is increasing evidence for methylene blue having a better safety profile $[42,43]$, but more work needs to be done on regimen, dose-finding and acceptability $[44,45]$. The group supported the further development of these drugs and considers it a priority to develop more compounds active against transmission stages for all species of malaria.

\section{The roadmap}

Three themes were identified that need to be addressed simultaneously. Firstly, there are evidence gaps for primaquine itself, secondly, the manufacture and supply of primaquine needs mapping and thirdly, efforts to search for a safe and effective alternative to primaquine need to be supported. A schematic of the roadmap is shown in Figure 7.

\section{Providing evidence of the efficacy and safety of primaquine}

A range of studies from phase 1-4 were proposed that would inform decisions on the efficacy and safety of primaquine. These are outlined below.

\section{Phase 1: Identification of the lowest dose for efficacy}

Ex vivo gametocytocidal/ infectivity assays: because the active metabolites of primaquine are currently unknown, the interpretation of in vitro assays with primaquine is complicated. A possible approach would be to use healthy volunteers treated with different doses of primaquine. The plasma (containing primaquine metabolites) of these individuals could be used in membrane feeding experiments with cultured parasites to demonstrate lack of infectivity in mosquitoes of different doses of primaquine and in combination with ACT.

There is no proven relationship between mosquito infectivity and gametocytocidal effects so this may need to be repeated with a variety of parasite lines and volunteers of different ethnic backgrounds.

It was noted that much needed pharmacokinetic studies could be performed during the same experiments as could studies evaluating the different partner ACT, other gametocytocidal drugs and drugs in common use 


\section{Intended Indications for the use of primaquine to interrupt malaria transmission}

- Mass Drug Administration (MDA)/ Mass screen and treat (MSAT): Community-wide treatment with (MSAT)* or without screening (MDA) of individuals for malaria elimination. This is unlikely to be a one-off intervention, a series of repeated rounds of MDA may be required for maximal effect and optimal coverage. However there are limitations to the total number of rounds achievable due to the potential for drug resistance, cost, community acceptability and logistics

- Mini-MDA/ MSAT*: An MDA/ MSAT that is not conducted community-wide, but is targeted to transmission hotspots, i.e., select geographical areas of higher transmission intensity

- Micro-MDA/ MSAT*: An MDA/ MSAT that is conducted in individual households associated with high transmission or recently-/actively-infected individuals

- Epidemic control: Treatment to control an epidemic and prevent people from exporting gametocytes to new regions.

- Response to drug resistance: Treatment in the setting of drug resistance, where killing gametocytes will reduce the spread of drug resistant strains.

- Addition to $1^{\text {st }}$ line antimalarial treatment of symptomatic individuals. Whilst the use of a $45 \mathrm{mg}$ dose of primaquine in addition to first-line antimalarial treatment is current policy in a number of settings, this should not be widely recommended until evidence demonstrates safety and efficacy. There may be special circumstances where this may be advantageous such as in areas of very low endemicity, as well as situations where antimalarial treatment is used for the prevention of reintroduction of infection e.g. treatment of migrants or where treatment should have a specific aim to contain the spread of resistant parasites.

*This indicates a derivative approach using screening of the target group or population with a highly sensitive parasite detection tool and treatment with an alternative ACT to the first-line ACT together with primaquine.

Figure 6 Intended indications for the use of primaquine to interrupt malaria transmission.

that may interact with primaquine (e.g. antiretrovirals and drugs for tuberculosis).

\section{Phase 2: Establish the safety and efficacy of the optimal dose of primaquine in relevant sub-groups}

- Efficacy of low dose primaquine to assess posttreatment infectivity using common endpoints (see below) in G6PD normal individuals. A dose-finding study is currently under way in Uganda (NCT01365598).

- Studies to confirm safety of low dose primaquine in G6PD deficient.

- hemizygous males with lowest doses (dose escalation studies)

- heterozygous females (dose escalation studies)
- individuals of a given phenotypic G6PD enzyme function level, to establish a relationship between G6PD enzyme function level and safety, a proposed threshold enzyme function being in the range $20-30 \%$.

- Confirm safety and efficacy in infected population of unselected G6PD status (timeline 3-4 years).

- If safety with G6PD deficiency remains a problem, field usable and reliable point of care tests to detect G6PD deficiency will be needed and the effect of not treating a proportion of the population on transmission reduction modeled.

- Programmes to map the geographical distribution of G6PD deficiency in countries targeted for primaquine deployment. This should include assessment of the range of enzyme function levels in the population. 


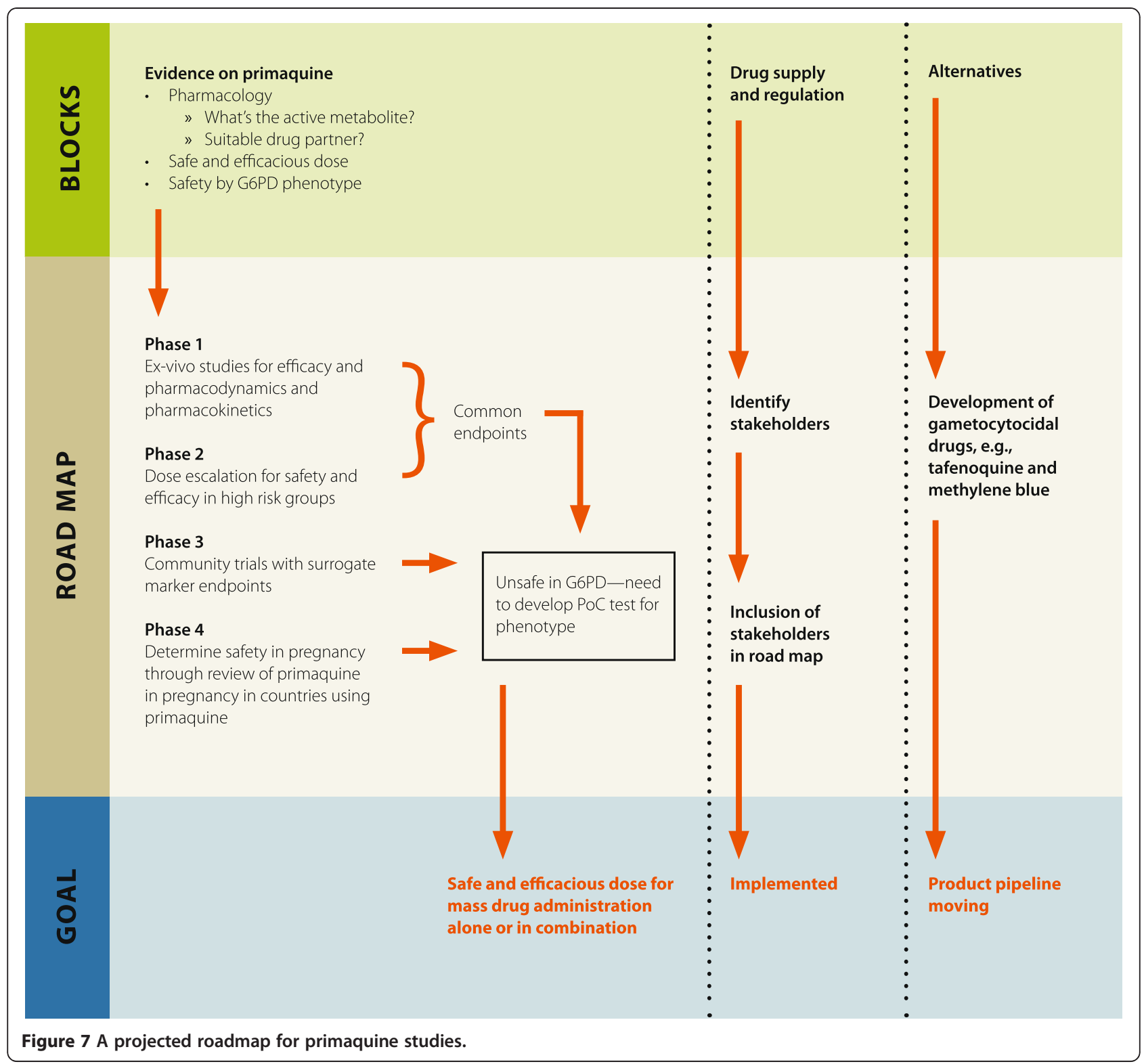

\section{Phase 3: Studies to establish utility at community level}

These may measure transmission reduction but may not necessarily need to be in the form of randomized controlled trials. Much can be learnt from the transmissionblocking vaccine field where designs such as a 'stepped wedge' design may be used with a focus on the indirect and community effects. Both prospective and retrospective pharmacovigilance studies will be needed and pregnancy registers will be an important component.

\section{Phase 4: Studies to review the safety in pregnancy}

Currently there is no evidence on safety of primaquine in pregnancy. Post-marketing surveillance is possible as several countries have adopted primaquine as policy, such as India, China and Sri Lanka. In these countries, pharmacovigilance could be supported to do a retrospective study following up women of reproductive age who have been treated with any dose of primaquine.

\section{Conclusion}

Primaquine may be a useful malaria control tool in lowendemic settings in Africa when used in combination with a blood schizontocide. For maximal effect it will need to be given to asymptomatic parasite carriers and therefore a safe and efficacious dose needs to be found that can be used in populations with G6PD deficiency. Studies designed to find this dose should contain common endpoints including infectiousness to mosquitoes seven days after treatment and gametocyte prevalence pre-treatment and seven days post-treatment to allow 
maximal comparability between trials. Safety endpoints need to be defined, particularly with regard to G6PD pheno- and genotype and pregnancy. Methylene blue and tafenoquine are alternative drugs but need further testing and establishing standard protocols could facilitate this process. Community trials should identify the added benefit of using primaquine in addition to a longacting ACT with the endpoint of community transmission reduction.

\begin{abstract}
Abbreviations
ACT: Artemisinin combination therapy; As: Artesunate; AUC: Area under the curve of gametocyte density over time; CQ: Chloroquine; EIR: Entomological inoculation rate; G6PD: Glucose-6-phosphate dehydrogenase; IRS: Indoor residual spraying; LLINs: Long-lasting insecticidal nets; MDA: Mass drug administration; PfPR: P. falciparum parasite prevalence; PQ: Primaquine; QT-NASBA: Quantitative real time nucleic acid sequence-based; SAE: Severe adverse event; SP: Sulphadoxine-pyrimethamine; WHO: World Health Organization.
\end{abstract}

\section{Competing interests}

The authors declare that they have no competing interests.

\section{Authors' contributions}

$A C E, R G$ and $C D$ wrote and edited the manuscript, $A C E, M H$ and JH were meeting rapporteurs and together with TB and LvS, contributed to the manuscript. All authors read and approved the final manuscript.

\section{Acknowledgements}

The authors are thankful for the positive and lively discussion of all the attendees who form the Primaquine in Africa Discussion Group. These were Salhiya Ali National Malaria Control Programme, Zanzibar), Keith Arnold (University Medical Schools, USA; Saigon University, Vietnam \& Chinese University, China), Ashenafi Assefa (Ministry of Health, Ethiopia), Kevin Baird (The Eijkman-Oxford Clinical Research Unit, Indonesia), Karen Barnes (Medical Research Council, South Africa), Teun Bousema (Radboud University Nijmegen Medical Centre, The Netherlands and London School of Hygiene and Tropical Medicine, UK), Janice Culpepper (Bill and Melinda Gates Foundation, USA), Karen Day (New York University, USA), Diadier Diallo (Institute de Recherche en Sciences de la Santé-Direction Régionale de I'Ouest [IRSS-DRO], Burkina Faso), Susan Dixon (GlaxoSmithKline, UK), Gonzalo Domingo (PATH, USA), Chris Drakeley (London School of Hygiene and Tropical Medicine, UK), Alice Chi Eziefula (London School of Hygiene and Tropical Medicine, UK), Roly Gosling (University of California, San Francisco, USA), Justin Green (GlaxoSmithKline, UK), Brian Greenwood (London School of Hygiene and Tropical Medicine, UK), Heiner Grueninger (Novartis, Switzerland), Rosalind Howes (University of Oxford, UK), Michelle Hsiang (University of California, San Francisco), Jimee Hwang (Centers for Disease Control and Prevention, USA), Patrick Kachur (Centers for Disease Control and Prevention, USA), Akira Kaneko (Osaka University Medical School, Japan and Karolinska University Hospital, Sweden), David McGibney (Medicines for Malaria Venture, Geneva), Olaf Mueller (Heidelberg University Hospital, Germany), Joseph Okebe (Medical Research Council, The Gambia and Prince Leopold Institute of Tropical Medicine, Belgium), Pascal Ringwald (World Health Organization, Geneva), Dennis Shanks (Army Malaria Institute, Australia), Erin Shutes (Bill and Melinda Gates Foundation, USA), Lorenz von Seidlein (Menzies School of Health Research, Australia), David Warhurst (London School of Hygiene and Tropical Medicine, UK). Umberto D'Alessandro (Prince Leopold Institute of Tropical Medicine, Belgium and Medical Research Council, The Gambia), Guoqiao Li (Saigon University, Vietnam \& Chinese University, China), Alan McGill, Lucy Okell (Imperial College London, UK) and Seif Shekelaghe (Ifakara Health Institute, Tanzania) were unable to attend but provided important contributions.

The authors would like to thank Dalia Iskander (London School of Hygiene and Tropical Medicine, UK) for logistical support and the Bill and Melinda Gates Foundation, The Global Health Group at the University of San Francisco in California and the Malaria Centre at the London School of Hygiene and Tropical Medicine for financial support. Alice C. Eziefula is funded by a Clinical Fellowship from the Wellcome Trust of Great Britain (\#090558/Z/09/Z).

\section{Author details}

${ }^{1}$ Malaria Centre, London School of Hygiene \& Tropical Medicine, Keppel St, London WC1E 7HT, UK. ${ }^{2}$ Global Health Group, University of California, San Francisco, 50 Beale Street, San Francisco, CA 94105, USA. ${ }^{3}$ Malaria Branch, Centers for Disease Control and Prevention, 1600 Clifton Rd, Atlanta, GA 30333, USA. ${ }^{4}$ Department of Pediatrics, University of California, San Francisco, San Francisco, CA 94143, USA. ${ }^{5}$ Menzies School of Health Research, Casuarina, NT 0811, Australia.

Received: 20 August 2012 Accepted: 2 October 2012 Published: 30 October 2012

\section{References}

1. World Health Organization: Guidelines for the treatment of malaria. 2nd edition. Geneva: WHO; 2011.

2. World Health Organization: World Malaria Report 2011. Geneva: WHO; 2011.

3. Clyde DF: Mass administration of an antimalarial drug combining 4aminoquinoline and 8-aminoquinoline in Tanganyika. Bull World Health Organ 1962, 27:203-212.

4. Abeyaratne KP, Halpe NL: Sensitivity to primaquine in Ceylonese children due to deficiency of erythrocytic glucose-6-phosphate dehydrogenase. Ceylon Med J 1968, 13:134-138.

5. Reeve PA, Toaliu H, Kaneko A, Hall JJ, Ganczakowski M: Acute intravascular haemolysis in Vanuatu following a single dose of primaquine in individuals with glucose-6-phosphate dehydrogenase deficiency. J Trop Med Hyg 1992, 95:349-351.

6. Khoo KK: The treatment of malaria in glucose-6-phosphate dehydrogenase deficient patients in Sabah. Ann Trop Med Parasitol 1981, 75:591-595.

7. Burgess RW, Bray RS: The effect of a single dose of primaquine on the gametocytes, gametogony and sporogony of Laverania falciparum. Bull World Health Organ 1961, 24:451-456.

8. Bousema T, Griffin JT, Sauerwein RW, Smith DL, Churcher TS, Takken W, Ghani A, Drakeley C, Gosling R: Hitting hotspots: spatial targeting of malaria for control and elimination. PLoS Med 2012, 9:e1001165.

9. Perine PL, Michael MT: A preliminary survey for glucose-6-phosphate dehydrogenase deficiency and haemoglobin S in Ethiopia. Ethiop Med J 1974, 12:179-184.

10. Mackerras MJ, Ercole QN: Some observations on the action of quinine, atebrin, and plasmoquine on Plasmodium vivax. Trans R Soc Trop Med Hyg 1949, 42:443-454.

11. Mackerras MJ, Ercole QN: Observations on the action of quinine, atebrin and plasmoquine on the gametocytes of Plasmodium falciparum. Trans $R$ Soc Trop Med Hyg 1949, 42:455-463.

12. Hill DR, Baird JK, Parise ME, Lewis LS, Ryan ET, Magill AJ: Primaquine: report from CDC expert meeting on malaria chemoprophylaxis I. Am I Trop Med Hyg 2006, 75:402-415.

13. Chemotherapy of malaria and resistance to antimalarials: Report of a WHO scientific group. World Health Organ Tech Rep Ser 1973, 529:1-121.

14. Gunders AE: The effect of a single dose of pyrimethamine and primaquine in combination upon gametocytes and sporogony of Laverania falcipara (Plasmodium falciparum) in Liberia. Bull World Health Organ 1961, 24:650-653.

15. Brewer GJ, Tarlov AR, Alving AS: Methaemoglobin reduction test: a new, simple, in vitro test for identifying primaquine-sensitivity. Bull World Health Organ 1960, 22:633-640.

16. Alving AS, Johnson CF, Tarlov AR, Brewer GJ, Kellermeyer RW, Carson PE: Mitigation of the haemolytic effect of primaquine and enhancement of its action against exoerythrocytic forms of the Chesson strain of Plasmodium vivax by intermittent regimens of drug administration: a preliminary report. Bull World Health Organ 1960, 22:621-631.

17. Hockwald RS, Arnold J, Clayman CB, Alving AS: Toxicity of primaquine in Negroes. J Am Med Assoc 1952, 149:1568-1570.

18. Weiss WR, Oloo AJ, Johnson A, Koech D, Hoffman SL: Daily primaquine is effective for prophylaxis against falciparum malaria in Kenya: comparison with mefloquine, doxycycline, and chloroquine plus proguanil. J Infect Dis 1995, 171:1569-1575. 
19. Baird JK, Surjadjaja C: Consideration of ethics in primaquine therapy against malaria transmission. Trends Parasitol 2011, 27:11-16.

20. Hodgkinson R, Courtney KO, Haggerty M: Effect of intermittent administration of a combination of amodiaquin and primaquine (Camoprim) on the hematocrit of primaquine-sensitive and nonsensitive children. Am J Trop Med Hyg 1961, 10:128-134.

21. Rieckmann KH, McNamara JV, Frischer H, Stockert TA, Carson PE, Powell RD: Gametocytocidal and sporontocidal effects of primaquine and of sulfadiazine with pyrimethamine in a chloroquine-resistant strain of Plasmodium falciparum. Bull World Health Organ 1968, 38:625-632.

22. Rieckmann KH, McNamara JV, Kass L, Powell RD: Gametocytocidal and sporontocidal effects of primaquine upon two strains of Plasmodium falciparum. Mil Med 1969, 134:802-819.

23. Shekalaghe S, Drakeley C, Gosling R, Ndaro A, van Meegeren M, Enevold A Alifrangis M, Mosha F, Sauerwein R, Bousema T: Primaquine clears submicroscopic Plasmodium falciparum gametocytes that persist after treatment with sulphadoxine-pyrimethamine and artesunate. PLoS One 2007, 2:e1023

24. Shekalaghe SA, Ter Braak R, Daou M, Kavishe R, van den Bijllaardt W, van den Bosch S, Koenderink JB, Luty AJ, Whitty CJ, Drakeley C, Sauerwein RW, Bousema T: Haemolysis after a single dose of primaquine coadministered with an artemisinin is not restricted to glucose-6phosphate dehydrogenase (G6PD A- variant) deficient individuals in Tanzania. Antimicrob Agents Chemother 2010, 54:1762-1768.

25. Song J, Socheat D, Tan B, Dara P, Deng C, Sokunthea S, Seila S, Ou F, Jian H, Li G: Rapid and effective malaria control in Cambodia through mass administration of artemisinin-piperaquine. Malar J 2010, 9:57.

26. von Seidlein L, Walraven G, Milligan PJ, Alexander N, Manneh F, Deen JL, Coleman R, Jawara M, Lindsay SW, Drakeley C, De Martin S, Olliaro P Bennett S, Schim Van Der Loeff M, Okunoye K, Targett GA, McAdam KP, Doherty JF, Greenwood BM, Pinder M: The effect of mass administration of sulfadoxine-pyrimethamine combined with artesunate on malaria incidence: a double-blind, community-randomized, placebo-controlled trial in The Gambia. Trans R Soc Trop Med Hyg 2003, 97:217-225.

27. Shekalaghe SA, Drakeley C, van den Bosch S, ter Braak R, van den Bijllaardt W, Mwanziva C, Semvua S, Masokoto A, Mosha F, Teelen K, Hermsen R, Okell L, Gosling R, Sauerwein R, Bousema T: A cluster-randomized trial of mass drug administration with a gametocytocidal drug combination to interrupt malaria transmission in a low endemic area in Tanzania. Malar 2011, 10:247.

28. Chen PQ, Li GQ, Guo XB, He KR, Fu YX, Fu LC, Song YZ: The infectivity of gametocytes of Plasmodium falciparum from patients treated with artemisinin. Chin Med J (Engl) 1994, 107:709-711.

29. Bousema T, Dinglasan RR, Morlais I, Gouagna LC, van Warmerdam T, Awono-Ambene PH, Bonnet S, Diallo M, Coulibaly M, Tchuinkam T, Mulder B, Targett G, Drakeley C, Sutherland C, Robert V, Doumbo O, Touré Y, Graves PM, Roeffen W, Sauerwein R, Birkett A, Locke E, Morin M, Wu Y, Churcher TS: Mosquito feeding assays to determine the infectiousness of naturally infected Plasmodium falciparum gametocyte carriers. PLoS One 2012, 7:e42821.

30. Schneider P, Bousema JT, Gouagna LC, Otieno S, van de Vegte-Bolmer M, Omar SA, Sauerwein RW: Submicroscopic Plasmodium falciparum gametocyte densities frequently result in mosquito infection. Am J Trop Med Hyg 2007, 76:470-474.

31. Bousema T, Drakeley C: Epidemiology and infectivity of Plasmodium falciparum and Plasmodium vivax gametocytes in relation to malaria control and elimination. Clin Microbiol Rev 2011, 24:377-410.

32. Okell LC, Ghani AC, Lyons E, Drakeley CJ: Submicroscopic infection in Plasmodium falciparum-endemic populations: a systematic review and meta-analysis. J Infect Dis 2009, 200:1509-1517.

33. Bousema T, Okell L, Shekalaghe S, Griffin JT, Omar S, Sawa P, Sutherland C, Sauerwein R, Ghani AC, Drakeley C: Revisiting the circulation time of Plasmodium falciparum gametocytes: molecular detection methods to estimate the duration of gametocyte carriage and the effect of gametocytocidal drugs. Malar J 2010, 9:136

34. Mendez F, Munoz A, Plowe CV: Use of area under the curve to characterize transmission potential after antimalarial treatment. Am J Trop Med Hyg 2006, 75:640-644.

35. Drakeley C, Sutherland C, Bousema JT, Sauerwein RW, Targett GA: The epidemiology of Plasmodium falciparum gametocytes: weapons of mass dispersion. Trends Parasitol 2006, 22:424-430.
36. Shanks GD, Oloo AJ, Aleman GM, Ohrt C, Klotz FW, Braitman D, Horton J, Brueckner R: A new primaquine analogue, tafenoquine (WR 238605), for prophylaxis against Plasmodium falciparum malaria. Clin Infect Dis 2001, 33:1968-1974

37. Edstein MD, Kocisko DA, Brewer TG, Walsh DS, Eamsila C, Charles BG: Population pharmacokinetics of the new antimalarial agent tafenoquine in Thai soldiers. Br J Clin Pharmacol 2001, 52:663-670.

38. Schneider P, Schoone G, Schallig H, Verhage D, Telgt D, Eling W, Sauerwein R: Quantification of Plasmodium falciparum gametocytes in differential stages of development by quantitative nucleic acid sequence-based amplification. Mol Biochem Parasitol 2004, 137:35-41.

39. Sinden RE, Carter R, Drakeley C, Leroy D: The biology of sexual development of Plasmodium: the design and implementation of transmission-blocking strategies. Malar J 2012, 11:70.

40. Okell LC, Griffin JT, Kleinschmidt I, Hollingsworth TD, Churcher TS, White MJ, Bousema T, Drakeley CJ, Ghani AC: The potential contribution of mass treatment to the control of Plasmodium falciparum malaria. PLoS One 2011, 6:e20179.

41. Kaneko A, Taleo G, Kalkoa M, Yamar S, Kobayakawa T, Bjorkman A: Malaria eradication on islands. Lancet 2000, 356:1560-1564.

42. Mandi G, Witte S, Meissner P, Coulibaly B, Mansmann U, Rengelshausen J, Schiek W, Jahn A, Sanon M, Wust K, Walter-Sack I, Mikus G, Burhenne J, Riedel KD, Schirmer H, Kouyaté B, Müller O: Safety of the combination of chloroquine and methylene blue in healthy adult men with G6PD deficiency from rural Burkina Faso. Trop Med Int Health 2005, 10:32-38.

43. Meissner PE, Mandi G, Witte S, Coulibaly B, Mansmann U, Rengelshausen J, Schiek W, Jahn A, Sanon M, Tapsoba T, Walter-Sack I, Mikus G, Burhenne J, Riedel KD, Schirmer H, Kouyaté B, Müller O: Safety of the methylene blue plus chloroquine combination in the treatment of uncomplicated falciparum malaria in young children of Burkina Faso [ISRCTN27290841]. Malar J 2005, 4:45.

44. Bountogo M, Zoungrana A, Coulibaly B, Klose C, Mansmann U, Mockenhaupt FP, Burhenne J, Mikus G, Walter-Sack I, Schirmer RH, Sié A, Meissner $P$, Müller O: Efficacy of methylene blue monotherapy in semiimmune adults with uncomplicated falciparum malaria: a controlled trial in Burkina Faso. Trop Med Int Health 2010, 15:713-717.

45. Coulibaly B, Zoungrana A, Mockenhaupt FP, Schirmer RH, Klose C, Mansmann U, Meissner PE, Muller O: Strong gametocytocidal effect of methylene blue-based combination therapy against falciparum malaria: a randomised controlled trial. PLoS One 2009, 4:e5318.

doi:10.1186/1475-2875-11-360

Cite this article as: Eziefula et al:: Rationale for short course primaquine in Africa to interrupt malaria transmission. Malaria Journal 2012 11:360.

\section{Submit your next manuscript to BioMed Central and take full advantage of:}

- Convenient online submission

- Thorough peer review

- No space constraints or color figure charges

- Immediate publication on acceptance

- Inclusion in PubMed, CAS, Scopus and Google Scholar

- Research which is freely available for redistribution 